\title{
Valor social de la Misión Milagro en el contexto venezolano
}

\author{
Social value of Miracle Mission in the venezuelan setting
}

\section{Ladys Marlene León Corrales}

Especialista de I Grado en Medicina General Integral. Misión Barrio Adentro. Mariara, Venezuela.

\begin{abstract}
RESUMEN
Datos estadísticos reflejan que en el mundo existen aproximadamente 45 millones de personas ciegas y su morbilidad aumenta significativamente. Venezuela no está ajena a estos problemas y las afecciones oftalmológicas han constituido uno de los problemas de salud de mayor magnitud. A partir de 1999 con la aprobación de la nueva Constitución Bolivariana se produce una revolución social, que define la salud como derecho del pueblo y responsabilidad del gobierno. Por la frecuencia con que se presentaban las enfermedades oftalmológicas y su repercusión económica y social surge la Misión Milagro como Alternativa Bolivariana para Las Américas destinada a tratar quirúrgicamente a personas afectadas de ceguera o deficiencia visual corregible. Desde el 2004 hasta la fecha han sido intervenidas quirúrgicamente más de 1000000 de personas afectadas por estas enfermedades. En la práctica médica asistencial se han constatado resultados satisfactorios en este programa. La Misión Milagro es una hazaña solidaria y humana sin precedentes en la historia mundial la cual tiene como esencia el carácter eminentemente social de la medicina cubana. La Misión Milagro en el contexto venezolano ha garantizado el diagnóstico y tratamiento oportuno y efectivo de un conjunto de enfermedades oftalmológicas en personas que históricamente fueron excluidas de la atención médica y apartadas de la vida social y productiva. Estas personas han recuperado su salud visual y logrado su reinserción al núcleo familiar, al trabajo y a la sociedad.
\end{abstract}

Palabras clave: Misión Milagro, Misión Barrio Adentro, Venezuela, medicina cubana. 


\begin{abstract}
Statistical data reveal that there are 45 blind people all over the world and their morbidity rises significantly. Venezuela is not free from these problems and the eye diseases represent one of the major health problems. After passing the new Bolivarian Constitution in 1999, there was a social revolution that defined health as a right of the people and governmental responsibility. Due to the frequency of eye diseases and their economic and social impact, the Miracle Mission emerged as part of the Bolivarian Alternative for the Americas aimed at surgically treating persons with blindness or any other visual problem that may be corrected. Since 2004 up to the present, more than one million persons have been operated on. In the medical assistance field, there have been satisfactory results of the program. The Miracle Mission is an unprecedented human solidarity deed in the world history, whose essence is the truly social nature of the Cuban medicine. The Miracle Mission has assured in the Venezuelan context the timely effective diagnosis and treatment of various eye diseases suffered by persons who had been excluded from the medical care service and isolated from the social and productive life of the country. These persons have recovered their visual health and they have reintegrated into their families, their workplaces and the society.
\end{abstract}

Key words: Miracle Mission, Barrio Adentro Mission, Venezuela, Cuban medicine.

\title{
I NTRODUCCI ÓN
}

Las alteraciones de la visión han constituido una de las principales preocupaciones del hombre pues es a través de este órgano que se percibe el $80 \%$ del mundo exterior. En el proceso de génesis de estas alteraciones influyen un grupo de factores socio-económicos que no solo participan en su desarrollo sino que inciden negativa o positivamente en su evolución.

Datos estadísticos reflejan que en el mundo existen aproximadamente 45 millones de personas ciegas y su morbilidad aumenta significativamente de 1- 2 millones por año, en las próximas dos décadas se duplicará su número, aún con la disponibilidad de intervenciones altamente rentables. La organización médica mundial asegura que las dos terceras partes de toda la ceguera pueden ser curadas o al menos prevenidas con el tratamiento adecuado. Cuatro de cada cinco personas perderán la visión innecesariamente. ${ }^{1}$

Muchas de las causas de ceguera evitable en países de bajo presupuesto están directamente relacionadas a la pobreza, incluyendo hambre, malnutrición y acceso limitado a la salud, educación, agua y servicios sanitarios. Estos problemas son los más agudos en las regiones menos desarrolladas económicamente, hogar de más del $90 \%$ de la gente con discapacidad visual, que vive atrapada en la pobreza, con la perspectiva de ser excluida de la atención sanitaria básica, educación, servicios sociales y sus consecuentes riesgos de aislamiento, enfermedades y exclusión económica. 
La deficiencias visuales conllevan profundas desventajas económicas a los individuos, sus familias y sociedades. Varios estudios recientes han cuantificado estos impactos, entre ellos el trabajo hecho por Frick y Foster referente al impacto de la ceguera evitable en la productividad económica global y un estudio del impacto económico de la cirugía de cataratas en los individuos. Ambos trabajos demuestran la magnitud de los retornos económicos y sociales de las inversiones en ceguera evitable. ${ }^{2}$

Fue en reconocimiento de este pronóstico inaceptable, que la Organización Mundial de la Salud (OMS) y la Agencia Internacional para la Prevención de la Ceguera (IAPB) [por sus siglas en Inglés], lanzaron en el año 1999 la iniciativa conjunta conocida como VISIÓN 2020: El Derecho a la Visión. Esto provee el marco programático para eliminar la ceguera evitable para el año $2020 .^{3}$

Han existido otros programas aislados en función de resolver este problema, en tanto la Organización Internacional Cristoffel-Blindenmission (CBM) ha realizado encuestas comunitarias en muchos países de América Latina para la determinación de la prevalencia de la ceguera por cataratas e indirectamente la estimación de otras causas. ${ }^{4}$

El programa ORBIS, organización humanitaria sin fines de lucro, tuvo su génesis en el año 1982, por iniciativa de un grupo de oftalmólogos de la Universidad de Indiana, Estados Unidos, con el fin de eliminar la ceguera, y hacer énfasis en la enseñanza del cuidado de la visión, tanto quirúrgica como preventivamente. Los especialistas del programa laboran en el interior de un avión DC-10, donde son trasladados el personal y el equipamiento. ${ }^{5}$

No es menos cierto que gracias a estas intervenciones, causas como la ceguera por tracoma, oncocercosis y la infantil por déficit de vitamina A, han ido disminuyendo. Sin embargo, han tenido un alcance limitado en respuesta a este fenómeno mundial, al continuar incrementándose el número de casos anual por catarata, glaucoma y retinopatía diabética, entre otras, que corresponden a alteraciones directamente relacionadas con el envejecimiento de la población, los cambios alimentarios y la desigualdad social. ${ }^{6}$

Venezuela no está ajena a estos problemas actuales, y las afecciones oftalmológicas han constituido uno de los problemas de salud de mayor magnitud sin contar con el respaldo de una política sanitaria ni estatal.

A partir de 1999 con la aprobación de la nueva Constitución Bolivariana de Venezuela se produce una revolución social, que define desde sus inicios la salud como derecho del pueblo y una responsabilidad del gobierno. Eso sin dudas, significó un salto cualitativo extraordinario, pero en los primeros años del proceso revolucionario, pese a muchos planes y esfuerzos, no se encontraba el verdadero camino para producir cambios en los servicios de salud.

Durante tres años se ensayaron vías coyunturales, que dieron respuesta a numerosos problemas, pero fue la experiencia de Barrio Adentro de la Misión de Colaboración Cubana, nacida en abril de 2003, la que abrió el sendero a la Medicina General Integral y permitió descubrir hacia dónde tenían que ir dirigidos los cambios. En virtud de un acuerdo de cooperación entre ambos países, comenzaron a laborar en los servicios de salud venezolanos los médicos cubanos, demostrando que era posible cambiar la vida de millones de seres humanos que crecieron sin asistencia médica y en la desesperanza. 
El ejemplo de los galenos, que comparten las esperanzas de 17 millones de excluidos fue la inspiración de dedicar todas las energías, recursos y tiempo, al noble proyecto de apoyar el nacimiento de un sistema de salud que tiene en la asistencia primaria, con los médicos en las comunidades, su base fundamental. Todo esto marcó el camino para que se desarrollaran acciones encaminadas a responder a las necesidades de salud de la población y contribuir a mejorar la calidad de vida del pueblo venezolano. ${ }^{7}$

En el año 2004 se realizó el primer estudio nacional de prevalencia de ceguera, que arrojó un 3,5\% de personas ciegas mayores de 50 años, la catarata fue la principal causa en el $65,7 \%$. Se detectaron otras causas como: glaucoma $15,0 \%$, errores refractivos $5,0 \%$, retinopatía diabética $2,9 \%$ y la degeneración macular asociada a la edad en el $2,9 \%{ }^{8}$

Por la frecuencia con que se presentan las enfermedades oftalmológicas y su repercusión económica y social surge la Misión Milagro como Alternativa Bolivariana para Las Américas (ALBA) destinada a tratar quirúrgicamente a personas afectadas de ceguera o deficiencia visual corregible, es reflejo del desarrollo científico técnico en función de la sociedad pues ha logrado devolverle la visión a un gran número de pacientes de muchos países de América Latina y El Caribe. Desde el 2004 hasta la fecha han sido intervenidas quirúrgicamente más de 1000000 de personas afectadas por estas enfermedades. En la práctica del desempeño asistencial se ha observado y constatado resultados satisfactorios en este programa. Todo ello como resultado del esfuerzo desplegado por los colaboradores de la Misión Barrio Adentro en las comunidades y por la Revolución Bolivariana para tratar de saldar la deuda social que existe en materia de salud con la población más excluida de Venezuela.

Sin dudas, la revolución social constituye un paradigma en la actualidad, que tiene como particularidad, ya demostrada en la Revolución Cubana, como el hacer la revolución social, conduce o facilita el desarrollo de la revolución científica técnica, ${ }^{9}$ y su esencia está sustentada en la formación de valores sociales. En el contexto venezolano este conjunto de valores sociales se enfatizan en la labor que han venido desarrollando los médicos y técnicos cubanos en Misión Milagro, en aras de garantizar una mejor calidad de vida de la población venezolana. El objetivo fundamental del presente estudio consiste en reflejar el valor social que posee esta Misión en el contexto venezolano.

\section{LA MISIÓN}

La relación del hombre con la realidad se produce a través de la práctica el conocimiento y la valoración.

Entre la valoración, el conocimiento y la práctica existe una relación indisoluble. El hombre en la medida que obtiene sus fines, valora su actividad y sus resultados, estableciendo una correlación entre estos últimos y los fines anteriormente propuestos.

La actividad valorativa se estudia a través de la axiología, la cual ocupa un importante lugar en la concepción filosófica del mundo.

El valor no es un fenómeno u objeto de la realidad en si mismo, sino una cualidad de los objetos adquirida en el curso de la práctica histórico-social. ${ }^{10}$ 
Los valores constituyen una función de los fenómenos y objetos consistentes en la posibilidad de servir de alguna forma a la actividad práctica de los hombres, por cuanto la práctica representa un proceso objetivo, los valores expresan las necesidades objetivas de la sociedad, surgen y existen independientemente de la voluntad de los hombres.

Fabelo Corzo en su obra refiere, por valor:

La capacidad que poseen determinados objetos y fenómenos de la realidad objetiva de satisfacer alguna necesidad humana, es decir, la determinación social de estos objetos y fenómenos consistentes en su función de servir a la actividad práctica de los hombres. ${ }^{11}$

El valor como fenómeno de la conciencia social orienta la actuación de los hombres, constituyen los sueños que orientan su quehacer y son una expresión ideológica de sus intereses. ${ }^{12}$

La Salud por ende, puede ser analizada a través de esta teoría de valores, al constituir un valor fundamental de la humanidad, que garantiza su existencia al satisfacer necesidades esenciales para ella, la calidad de su vida, y su grado de adaptación al medio natural y social, así como su carácter sistémico, estrechamente relacionado con su modo de vida y las concepciones imbricados entre sí.

En la sociedad se han ido desarrollando instituciones y organizaciones que son decisivas a la hora de conformar las acciones que despliega el hombre en el campo de la salud, la política de salud constituye un elemento fundamental en todas estas acciones y se manifiesta a través del Sistema de Salud imperante. Las trayectorias que siguen la ciencia y la tecnología médica están determinadas por un entramado de fuerzas sociales, que sirven de espacios donde operan determinaciones políticas e ideológicas en su constitución.

En Cuba, después del triunfo revolucionario el estado puso énfasis en la educación médica, y la medicina cubana tiene un carácter socio-preventivo- curativo, o sea, el perfil de salida de los médicos cubanos es hacia la Medicina General Básica y la Medicina General Integral, con la finalidad de promover salud en la comunidad, prevenir enfermedades, rehabilitar pacientes y no sólo curar enfermos. ${ }^{13}$

Por otra parte, no sólo existe una voluntad política, sino una cultura política, fundamentada en ideales y valores señalados, pero ante todo expresada en relación con el conocimiento científico.

El Sistema de Salud cubano se desarrolla bajo los imperativos de la lucha política, especialmente externa, el cual responde a una estrategia que combina el desarrollo de alta tecnología, la organización social y pesquisaje precoz masivo con el sustento de la participación comunitaria.

En este Sistema se aprecia una simbiosis entre la matriz biomédica y la social de la única medicina que ofrece posibilidades de desarrollo en ambos planos, en el de la manifestación clínica en su forma individualizada, y en el de la Salud Pública con su fundamentación epidemiológica y el reconocimiento de las necesidades sociales de salud. "Se vislumbra la posibilidad de fusionar la biotecnología, y la inmunología avanzada con el desarrollo del nivel primario de atención de salud y la medicina poblacional". El Dr. Agustín Lage afirma que esa síntesis puede ser un aporte de carácter mundial. Pueden darse relaciones inéditas entre lo clínico y lo epidemiológico. 
La política cubana de salud como elemento de la política social y científica está generando oportunidades de establecer una dinámica alternativa de la exploración del conocimiento en salud, más cercana a las necesidades y los valores sociales contextuales. ${ }^{12}$

Cuba puede mostrar realidades de un modo de producción de conocimientos basado en valores sociales, dirigidos a manifestar objetivos e intereses comunes que lejos de resaltar la vulnerabilidad del sistema político conduce a fortalecer la identidad cultural y también explotar sus ventajas competitivas. En sus últimas intervenciones sobre la salud, Fidel Castro resaltó la idea de continuar aspirando a una excelencia en los servicios. Sin dudas aspiración necesaria y posible.

Los centros de educación superior tienen un gran reto para garantizar el carácter de integración en el desarrollo de la labor educativa y político-ideológica, pues no se debe ubicar en un primer plano la formación y desarrollo de conocimientos, hábitos y habilidades en los estudiantes, sino también los valores como componente clave de la personalidad. El médico cubano es un genuino representante de los principios de la ética médica, y por tanto refleja los valores y cualidades morales de la profesión basado en ellos, porque lo siente como necesidad y no porque esté normado; máxime en los momentos actuales donde los avances de la ciencia y la técnica van perfeccionando cada vez más la actividad médica y se requiere de un equilibrio entre las nuevas tecnologías y el carácter humanista de la profesión. ${ }^{13}$

Lo anterior demuestra el carácter altamente humano, justo y equitativo del sistema de salud, que va más allá de la formación de un profesional con una concepción salubrista y la utilización de la revolución científico-técnica en función de esta, sino en la formación de valores como el altruismo e internacionalismo.

El Programa Integral de Atención para la Salud dirigido a países del tercer mundo ha abierto las posibilidades de brindar solidaridad e investigar sus principales problemas de salud los cuales se insertan en una política, permite la generación de formas alternativas a la medicina, la investigación y la industria farmacéutica, como enfrentamiento al subdesarrollo. En ese sentido no sólo tiene significación simbólica, es "una manera de hacer", constituye una mentalidad, científica, amplia y flexible que contribuye a la asimilación de conocimientos y técnicas acumuladas por la tradición social, proveniente de numerosas culturas e incluidas en el nivel cotidiano para convertirse en científica. ${ }^{13}$

Desde inicios de los años 60, los profesionales cubanos de la salud brindan su colaboración humanitaria en varios países del mundo, África, Asia y América Latina, imbuidos en el proceso de globalización de la economía, donde los ricos son cada vez más ricos y los pobres son cada vez más pobres y más numerosos. Venezuela, no escapa de esta realidad, al inicio del triunfo de la revolución bolivariana, el sistema de salud se encontraba fraccionado, caracterizado por la medicina elitista que identifica al modelo de salud capitalista y que distaba mucho de ser accesible, por lo que el cuadro de salud social se encontraba en franco deterioro. Al irrumpir el proceso revolucionario en 1999 se inicia un cambio de la política, jurídica, económica y social para poner en marcha un nuevo modelo de desarrollo, donde prime el sistema democrático, con una economía eficiente en medio de la globalización.

Se fusiona el Ministerio de Sanidad y Asistencia Social con el Ministerio de la Familia, creándose el Ministerio de Salud y Desarrollo Social (MSDS). Para ese entonces la tasa de médicos por 10000 habitantes representaba sólo el 19,7\% del total de médicos del país. ${ }^{14}$ 
La Tragedia de Vargas, como se le conoce al conjunto de deslaves, corrimientos de tierras e inundaciones ocurridas en las costas caribeñas de Venezuela, en diciembre de 1999, provocó un cuadro epidemiológico deficiente. Ante tales circunstancias la máxima dirección de Cuba, ofreció en nombre del pueblo cubano su acostumbrada ayuda solidaria, y es entonces cuando arriban a la patria de Bolívar los primeros galenos del Programa Integral de Salud, que inician su labor en los cerros de Caracas. En el 2003 se extiende la Misión Barrio Adentro en todo el país, como "Madre" de las misiones en América Latina y otros países. Comienza la prestación de servicios de salud a una población excluida y desasistida hasta la fecha, por el arraigo de un sistema capitalista que otrora gobernaba sus destinos. Con el decursar del tiempo se hacía necesario aumentar el poder de resolutividad del médico de asistencia para ofrecer un servicio más completo y de mejor calidad, surgen los Centros Diagnósticos Integrales, las Salas de Rehabilitación Integral, los Centros Médicos de Alta Tecnología. Este sistema permitió identificar la verdadera situación de salud del pueblo venezolano donde las enfermedades oftalmológicas representaban una incidencia significativa y afectaban la calidad de vida de la población y los escasos recursos económicos con que contaban gran parte de los enfermos, en su mayoría pertenecientes al grupo de excluidos del país. A partir de entonces aparecen los programas sociales, como la Misión Milagro en Julio de 2004.

Esta Misión se inicia con miles de venezolanos que viajan al archipiélago cubano para recuperar la calidad de su visión, como parte de la gestión del Presidente Hugo Chávez Frías, en el marco de la Alternativa Bolivariana de Las Américas (ALBA), en su afán de generar soluciones a uno de los problemas que afecta a millones de personas en el mundo, la ceguera prevenible y la baja visión, que pueden ser corregidas quirúrgicamente, irrumpiendo como un novedoso modelo profesional en el campo de la Oftalmología y que cumple los principios de accesibilidad y equidad para el desarrollo de la Salud Visual, con la utilización de alta tecnología que permitiera elevar los indicadores de la calidad de vida de la población de todas las latitudes de la geografía venezolana.

Por acuerdos entre ambos gobiernos, en el primer trimestre del año 2006 se decide instalar un escenario de las intervenciones quirúrgicas en varios estados de Venezuela dentro de los cuales se escoge Carabobo para asegurar la salud visual de esta población y de otros estados colindantes. Se inaugura entonces el Centro Oftalmológico "Simón Bolívar" del estado de Carabobo el 17 de julio de ese mismo año, el cual cuenta con tres posiciones quirúrgicas y todo el equipamiento de alta tecnología, así como el personal especializado que permitió que en menos de 72 horas se estuvieran operando los primeros pacientes. Su misión está centrada en garantizar, el diagnóstico y tratamiento oportuno y efectivo de un conjunto de enfermedades oftalmológicas en personas que históricamente fueron excluidas de la atención y apartadas de la vida social y productiva por padecer de un problema solucionable y lograr la recuperación de su salud visual y su reinserción al núcleo familiar, al trabajo y a la sociedad.

Es el centro de referencia de la oftalmología cubana en Venezuela, hacia donde confluyen los pacientes de los estados colindantes de Aragua, Cojedes, Yaracuy y Carabobo con una población total de 4760840 habitantes.

Se encuentra equipado con tecnología de punta en la especialidad, entre los que se pueden mencionar el láser del segmento anterior que brinda la posibilidad de realizar capsulotomía, membranotomía, pupiloplastia, barrido del lente, iridectomía, otros como la ultrasonografía ocular de retina clínica, o novedosos métodos quirúrgicos como las microcirugías en general: extracción extracapsular del cristalino, facoemulsificación, los lentes intra oculares, que permiten la visón inmediata, todo lo cual posibilita el diagnóstico y tratamiento de las enfermedades 
oftalmológicas y que desecha viejos mitos asistenciales como la larga estadía de los pacientes.

Los pacientes atendidos en el Centro Oftalmológico provienen del pesquisaje realizado por los médicos cubanos, especialistas y residentes en oftalmología que se encuentran laborando en las comunidades, muchas de ellas en lugares de difícil acceso, en aras del principal pilar que es el ser humano y donde se extiende la atención de la salud y la entrega de medicamentos a toda la población. Estos pacientes pertenecen a los sectores más pobres de la población venezolana, aunque no son excluidos aquellos de otra clase social y/o pensamiento político.

Este programa genera una combinación de tecnología de avanzada en medios diagnósticos y quirúrgicos con una tecnología de organización social que canaliza la atención primaria hacia niveles de atención especializados, como resultado de los pesquisajes masivos ha permitido detectar las enfermedades quirúrgicas que a posteriori serían resueltas por la Misión Barrio Adentro. ${ }^{15}$ En Carabobo han sido intervenidos quirúrgicamente más de 53000 pacientes de estas enfermedades, lo que demuestra como la Ciencia y la Tecnología cuando se utilizan por parte de los gobiernos en función de darle solución a problemas de salud de la población adquiere un componente valorativo positivo que favorece el desarrollo social del país. Lo cual pone en evidencia el cumplimiento del principio ético rector Primun non nocere, de la medicina cubana y a su vez constituye el basamento sobre el cuál los profesionales de la salud cubana manifiestan su labor diaria, sustentados en valores sociales y principios fundamentales de la salud.

\section{Valores que caracterizan a los profesionales cubanos}

Humanismo médico. Ser benevolente, siempre hacer el bien, dedicando todos los esfuerzos y conocimientos científicos y técnicos a la prevención, recuperación, rehabilitación y promoción de la salud humana.

Gran sensibilidad ante los problemas de los pacientes y sus familiares, ayudarlos a resolverlos. Amor hacia los demás y hacia la profesión, ser íntegro, piadoso. Identificarse con el ser humano independientemente de su posición, ejercer la profesión con altruismo, ver al hombre como el fin supremo de la actividad médica y no el medio. Rechazo a la maleficencia, al trato desigual a los pacientes, a la injusticia, oposición al egoísmo.

Profesionalidad médica. Hacer sagrada la carrera, entrega a la profesión, demostrar preparación médica especial, dominio de la técnica y la tecnología de avanzada e interés por la investigación.

Respetarse a sí mismo y respetar los derechos de pacientes, familiares, colegas y demás personas.

Salvaguardar las confidencias de los pacientes aún después de fallecidos. Mantener estándares elevados de conducta profesional, con dominio pleno de la comunicación y del trabajo en equipo.

Mantener incólumes por todos los medios el honor y las nobles tradiciones de la profesión médica.

Responsabilidad. Compromiso permanente, personal y voluntario del médico con el paciente y con las demás tareas, manifestado en la actuación correcta en el 
cumplimiento cabal de sus obligaciones, sin que esto limite su autenticidad y creatividad en el desempeño de su profesión.

Sencillez. Estilo desprovisto de artificios, moderación en las relaciones interpersonales, con gran sentido de humildad, sin que decrezca el médico, sino que lo eleve, no creerse por encima de los demás.

Honestidad. Demostración de un sincero interés por el paciente, valorándolos por igual, independientemente de las posibilidades económicas, parentesco y relación personal; rechazo al soborno y a la mentira, actuando de buena fe, con justeza. Congruencia entre el discurso y el hacer.

Cortesía. Aspecto y porte personal correctos, buenos modales, uso de un lenguaje apropiado, delicadeza, buen trato, buen tacto, alto nivel de educación formal. Rechazo a los actos de grosería y maltrato.

Horizonte cultural. Tener una visión general del desarrollo de la humanidad en el más amplio sentido, así como de las diferentes manifestaciones artísticas y literarias. Ser sensible de la naturaleza y la obra humana. Comportarse en su relación con el medio y con las demás personas de acuerdo con su alto nivel de desarrollo intelectual.

Internacionalismo. Identificación con la política internacionalista de la Revolución Cubana y con las causas justas de otros pueblos, prestar o aceptar ayuda desinteresada a cada uno de estos, en cualquier forma o establecer intercambios mutuamente ventajoso

\section{Principios fundamentales de la salud cubana}

Equidad. Permite el acceso equitativo de las oportunidades, donde todos los grupos de la sociedad tienen los mismos derechos a la salud, incluyendo el acceso económico y geográfico a los servicios.

Gratuidad. Recibir los servicios de salud sin recibir valor monetario.

Solidaridad. La relación médico-paciente es sustentada bajo este principio, a través de ella se instaura una nueva forma de comunicación entre los seres humanos.

Accesibilidad. Posibilidad de ser atendidos en los servicios de salud. Existen tres tipos: material (distancia, transporte, horarios), económicas (costos de viaje, de los honorarios) y sociales o culturales (barreras por pautas culturales, del idioma, y otras).

Universalidad. La salud pública tiene que ver con todas las personas, ya sean sanas o enfermas, reciban el servicio o no, pertenezcan a determinado género, edad o grupo étnico e incluso ignoren casi todo acerca de sus posibilidades.

Corresponsabilidad. Es un acto de responsabilidad y un deber social.

Justicia. Los servicios brindados deben ser asequibles a todos sin excepción de raza, clase social, sexo o edad.

La Misión Milagro es una hazaña solidaria y humana sin precedentes en la historia mundial la cual tiene como esencia el carácter eminentemente social de la medicina 
cubana, que no sólo ve al hombre en su proceso de salud-enfermedad sino que se mueve desde el análisis de calidad de los procesos biológicos y la vida psicosocial del individuo hasta la calidad de las condiciones de vida de la sociedad, por otra parte, es un programa que permite no sólo la labor asistencial sino que desarrolla programas formativos de esta especialidad, maestrías, doctorado en ciencias de la salud, y perfeccionamiento del capital humano profesando un mejor futuro, utilizando la investigación, el amplio uso de la tecnología más novedosa y la estrecha vinculación de la teoría con la práctica, para garantizar una atención médica con calidad y excelencia y consolidar la triada entre la asistencia, la docencia y la investigación, que constituye una fortaleza no sólo al contar con un personal especializado en cada área, sino que alcanza una dimensión tal que se ha extendido a Bolivia, China y otros países, como expresión del internacionalismo.

Se puede concluir que los principios de la ética médica de los oftalmólogos y técnicos cubanos en Misión Milagro se corresponden con los de la medicina cubana. La Operación Milagro como manifestación particular de la Misión Médica Cubana en Venezuela representa el paradigma médico social que sustenta la medicina cubana en el mundo. La política de salud que se desarrolla en Barrio Adentro, permite elevar los índices de desarrollo social en Venezuela al aplicar el desarrollo científico técnico en función de la satisfacción de la población. La Misión Milagro en el contexto venezolano ha traído como resultado elevar los indicadores que permiten alcanzar elevado índice de desarrollo humano.

\section{REFERENCI AS BI BLI OGRÁFI CAS}

1. Organización Mundial de la Salud. Programa de Prevención de la ceguera evitable: Visión 2020. Ginebra: OMS;2001.

2. Frick KD, Foster A. La magnitud y costo de la Ceguera Global: un problema creciente que puede ser aliviado. Amer J Ophthal. 2003;135(4):471-7.

3. Organización de Naciones Unidas. Ceguera, pobreza y desarrollo: el impacto del Programa Visión 2020 en las metas del milenio. New York: ONU; 2004.

4. Organización Mundial de la Salud. Estrategias para la Prevención de la Ceguera en los Programas Nacionales. Un enfoque desde el punto de vista de la atención primaria de salud. 2da ed. Ginebra: OMS; 1998.

5. Proyecto ORBIS [sitio en Internet]. [actualizado 26 May 2003; citado 9 Jul 2008]. Disponible en: http://www.nnc.cubaweb.cu

6. Organización Mundial de la Salud. Eliminación de la ceguera evitable. Informe de la 56 Asamblea Mundial de la Salud. Punto 14.17 del orden del día; 2003 May

28[sitio en Internet]. [actualizado 24 Feb 2004; citado 9 Jul 2008]. Disponible en: http://publications. paho.org/spanish/moreinfo.cfm

7. Ministerio para el Poder Popular de Venezuela. Comunicación e Información. En: Colectivo de autores, editores. Barrio adentro una obra de corazón. Caracas: Imprenta Nacional; 2008.

8. Siso F, Esche G, Limburg H. Test Nacional de Catarata y Servicios Quirúrgicos. Primera Encuesta Nacional. Rev Oftalmol Venezuela. 2004;61(2): 112-39. 
9. Ramonet I. Milagros en Cuba. Juventud Rebelde. 15 de Oct de 2004.

10. Prieto Ramirez D, Aguirre del Busto R. La salud como valor social. En: Colectivo de autores, editores. Lecturas de Filosofía, Sociedad y Salud. La Habana: Editorial Ciencias Médicas; 2000.

11. Fabelo Corzo JR. Práctica, Conocimiento y Valoración. La Habana: Editorial Ciencias Sociales; 1989.

12. Pérez Sánchez AM, Martín Linares X. Educación en valores en el profesional de Ciencias Médicas. Rev Cubana Salud Pública [serie en Internet]. 2003[citado 9 Jul 2008]; 1(29). Disponible en:

http://scielo.sld.cu/scielo.php?script=sci_arttext\&pid=S0864-

$\underline{34662003000100010 \& \operatorname{lng}=\mathrm{es} \& \mathrm{n} r \mathrm{~m}=\mathrm{iso}}$

13. Seijo Echevarría BM, Cardoso Pérez R, Álvarez Vázquez J, Valle Sánchez T, Muñoz Rodríguez M. Los valores ético profesionales del médico: su diagnóstico. Rev Humanidades Médicas. 2001;1(2).

14. Macías Llanes ME. La política de Salud Cubana y los valores sociales a la luz de los Estudios de Ciencia-Tecnología-Sociedad. Rev Humanidades Médicas. 2005; 5(13).

15. Ciencia y Técnica [sitio en Internet]. [actualizado 23 Nov 2003; citado 28 Jul 2008]. Disponible en: http://www.wikipedia.es

Recibido: 17 de marzo de 2009.

Aprobado: 28 de marzo de 2009.

Ladys Marlene León Corrales. Misión Barrio Adentro. Mariara, Venezuela. 